C-A/AP/\#192

February 2005

\title{
Modeling Injection Trajectories on the Midplane of the C5 Dipole in Booster
}

\author{
C.J. Gardner
}

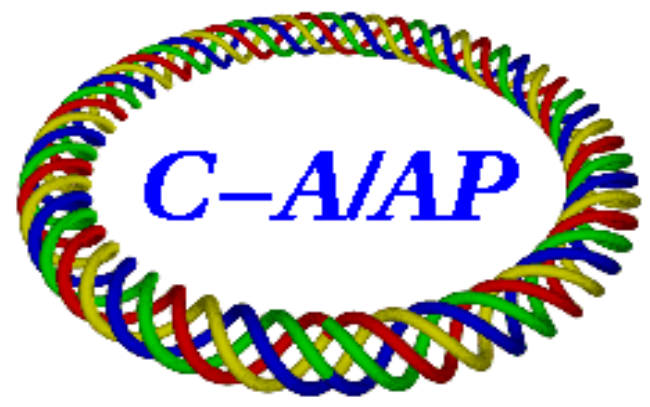

Collider-Accelerator Department Brookhaven National Laboratory

Upton, NY 11973 


\title{
Modeling Injection Trajectories on the Midplane of the C5 Dipole in Booster
}

\author{
C.J. Gardner
}

February 2, 2005

\section{The Booster Dipole Lamination}

A scale drawing of the Booster dipole lamination is shown in Figure 1. Here the pole width is $254 \mathrm{~mm}$. The distance from the pole to the backleg is $121 \mathrm{~mm}$. The width of the backleg is $133 \mathrm{~mm}$. The vertical gap between the poles is $82.55 \mathrm{~mm}$. The shims on the poles are $19 \mathrm{~mm}$ wide and the vertical gap between them is $77.75 \mathrm{~mm}$. The height of the lamination is $596.55 \mathrm{~mm}$ and the height of the H-shaped opening is $330.55 \mathrm{~mm}$. The brown rectangles indicate magnet coils. G10 insulating strips are indicated by the thin green rectangles. The thin pink rectangles indicate trim windings.

\section{Top View of the Dipole}

A top view of the Booster dipole is shown in Figure 2. Here the beam direction is from left to right. The horizontal and vertical axes give the $x$ and $y$ coordinates on the magnet midplane. The units are meters. The radial and azimuthal coordinates are defined by

$$
x=r \sin \theta, \quad y=r \cos \theta .
$$

The line $x=0$ bisects the magnet. The magnetic length is

$$
L=2.42 \mathrm{~m}
$$

and the bend angle is

$$
\theta_{B}=2 \pi / 36
$$


The dotted black curve is the nominal trajectory of circulating beam on the midplane; it is a circular arc with radius-of-curvature

$$
\rho_{0}=L / \theta_{B}=13.8656 \mathrm{~m}
$$

The center of curvature is the point $x=0, y=0$. As noted by Bleser [1] the centerline of the dipole is actually displaced radially outward by 0.18 $\mathrm{mm}$ with respect to the nominal trajectory. The practical consequences of this are negligible and we shall assume that the centerline and nominal trajectory coincide. The red lines and curves show the perimeter of the magnet iron. The left and right edges of the magnet iron lie on the lines

$$
\theta=\mp \theta_{2} / 2
$$

where $\theta_{2}=9.5763$ degrees as given by Bleser [1]. The gray curves are the projections of the walls of the vacuum chamber onto the midplane; these are $82.55 \mathrm{~mm}$ from the magnet centerline. The black curves are the projections of the pole and backleg boundaries. Those closest to the centerline mark the pole boundaries; they are $127 \mathrm{~mm}$ from the centerline. The outer black curves mark the backleg boundaries; these are $248 \mathrm{~mm}$ from the centerline.

\section{Magnetic Field on the Midplane}

Using the dimensions of the lamination, Wuzheng Meng has calculated the field on the dipole midplane. This was done using the Opera code with the assumption that the field is independent of azimuthal coordinate $\theta$. A plot of the field as a function of radius is shown in Figure 3. Here the blue curve gives the field in units of the field $B_{c}$ at the center of the magnet. The horizontal axis gives the radial distance from the projection of the pole edge on the midplane. The units are $\mathrm{mm}$. The black lines at 0 and 121 mark the pole and backleg edges. The dotted line at $-127 \mathrm{~mm}$ marks the center of the magnet; the red line at $-44.45 \mathrm{~mm}$ marks the vacuum chamber wall. Numerical integration of the equations of motion using this field gives the trajectory of the $\mathrm{H}$-minus beam on the midplane of the $\mathrm{C} 5$ dipole. 
Following is a list of the field data obtained by Meng. The first number in each pair is the radial distance from the magnet centerline in $\mathrm{cm}$; the second number is the midplane field in Gauss.

\section{Magnetic Field on Midplane of Booster Dipole}

0.01550 .32809468678

0.11550 .32816031908

$0.2 \quad 1550.32822595138$

$0.3 \quad 1550.32829158368$

$0.4 \quad 1550.32837187513$

$0.5 \quad 1550.32850314418$

$0.6 \quad 1550.32863441322$

$0.7 \quad 1550.32876568227$

$0.8 \quad 1550.32893649864$

$0.9 \quad 1550.32919026212$

$1.0 \quad 1550.32944402560$

$1.1 \quad 1550.32969778907$

$1.2 \quad 1550.33001257114$

1.31550 .33047072633

$1.4 \quad 1550.33092888151$

$\begin{array}{ll}1.5 & 1550.33138703670\end{array}$

$\begin{array}{ll}1.6 & 1550.33187868078\end{array}$

$1.7 \quad 1550.33255950149$

$\begin{array}{ll}1.8 & 1550.33324032219\end{array}$

$1.9 \quad 1550.33392114290$

2.01550 .33460196361

2.11550 .33559121098

$2.2 \quad 1550.33662313995$

$2.3 \quad 1550.33765506892$

$2.4 \quad 1550.33868699790$

$\begin{array}{ll}2.5 & 1550.33997207344\end{array}$

$2.6 \quad 1550.34152849548$

$2.7 \quad 1550.34308491751$

$2.8 \quad 1550.34464133954$

$\begin{array}{lll}2.9 & 1550.34619776158\end{array}$

$3.0 \quad 1550.34844962396$
3.11550 .35072817815

$3.2 \quad 1550.35300673234$

$3.3 \quad 1550.35528528653$

$3.4 \quad 1550.35786153424$

$\begin{array}{ll}3.5 & 1550.36107154500\end{array}$

$3.6 \quad 1550.36428155576$

$3.7 \quad 1550.36749156652$

$3.8 \quad 1550.37070157728$

$3.9 \quad 1550.37491285293$

$\begin{array}{ll}4.0 & 1550.37993564101\end{array}$

$4.1 \quad 1550.38495842908$

$4.2 \quad 1550.38998121716$

4.31550 .39500400523

$4.4 \quad 1550.40134445368$

$\begin{array}{lll}4.5 & 1550.40835992284\end{array}$

$4.6 \quad 1550.41537539201$

$\begin{array}{ll}4.7 & 1550.42239086117\end{array}$

$4.8 \quad 1550.42940633034$

4.91550 .43806376965

$\begin{array}{lll}5.0 & 1550.44761966103\end{array}$

$5.1 \quad 1550.45717555242$

$\begin{array}{ll}5.2 & 1550.46673144380\end{array}$

$\begin{array}{ll}5.3 & 1550.47628733519\end{array}$

$5.4 \quad 1550.48724719141$

$\begin{array}{lll}5.5 & 1550.49956897096\end{array}$

$\begin{array}{lll}5.6 & 1550.51189075051\end{array}$

$5.7 \quad 1550.52421253006$

$5.8 \quad 1550.53653430961$

$\begin{array}{lll}5.9 & 1550.54941405542\end{array}$

6.01550 .56401289975
6.11550 .57861174407

$6.2 \quad 1550.59321058839$

6.31550 .60780943272

$6.4 \quad 1550.62240827704$

$\begin{array}{lll}6.5 & 1550.63387149466\end{array}$

$\begin{array}{ll}6.6 & 1550.64481852892\end{array}$

6.71550 .65576556318

6.81550 .66671259745

$\begin{array}{ll}6.9 & 1550.67765963171\end{array}$

$\begin{array}{ll}7.0 & 1550.68440038160\end{array}$

$7.1 \quad 1550.68327556832$

$7.2 \quad 1550.68215075504$

$7.3 \quad 1550.68102594175$

$7.4 \quad 1550.67990112847$

$7.5 \quad 1550.67877631519$

$\begin{array}{ll}7.6 & 1550.62770798804\end{array}$

$\begin{array}{ll}7.7 & 1550.55667344091\end{array}$

$\begin{array}{ll}7.8 & 1550.48563889377\end{array}$

$7.9 \quad 1550.41460434663$

$8.0 \quad 1550.34356979950$

$8.1 \quad 1550.27253525236$

$8.2 \quad 1550.07280072485$

$8.3 \quad 1549.86721841522$

$8.4 \quad 1549.66163610558$

$8.5 \quad 1549.45605379595$

$8.6 \quad 1549.25047148631$

$8.7 \quad 1549.02405205726$

$8.8 \quad 1548.54008471874$

$8.9 \quad 1548.05611738022$

9.01547 .57215004169 
Note that a proton with $200 \mathrm{MeV}$ kinetic energy has magnetic rigidity $B \rho=2.149636 \mathrm{Tm}$. In order for it to follow a circular arc with nominal radius-of-curvature $\rho=\rho_{0}=13.8656 \mathrm{~m}$, the magnetic field must be 1550.34 Gauss.

\section{Magnetic Field on Midplane of Booster Dipole}

$9.1 \quad 1547.08818270317$

9.21546 .60421536465

9.31546 .06496402176

9.41544 .78367915136

$9.5 \quad 1543.50239428096$

$9.6 \quad 1542.22110941056$

$9.7 \quad 1540.93982454016$

9.81539 .65853966976

$9.9 \quad 1538.37725479937$

10.01535 .84895174343

$10.1 \quad 1533.24105178204$

10.21530 .63315182064

$10.3 \quad 1528.02525185925$

10.41525 .41735189785

10.51522 .80945193646

10.61518 .63779148480

10.71513 .75313684671

10.81508 .86848220861

10.91503 .98382757052

$11.0 \quad 1499.09917293242$

$11.1 \quad 1494.21451829432$

11.21488 .52758034307

11.31481 .05366214793

11.41473 .57974395278

11.51466 .10582575763

11.61458 .63190756248

$11.7 \quad 1451.15798936733$

11.81443 .68407117218

11.91432 .68681220579

12.01420 .85791569228
$12.1 \quad 1409.02901917878$

$12.2 \quad 1397.20012266527$

$12.3 \quad 1385.37122615176$

12.41373 .54232963826

12.51361 .18650442872

$12.6 \quad 1346.50007702568$

$12.7 \quad 1331.81364962265$

$12.8 \quad 1317.12722221961$

$12.9 \quad 1302.44079481657$

13.01287 .75436741353

13.11273 .06794001050

13.21257 .35441810100

13.31240 .31193161505

13.41223 .26944512909

$13.5 \quad 1206.22695864314$

$13.6 \quad 1189.18447215718$

13.71172 .14198567123

13.81155 .09949918528

13.91137 .59438019233

14.01119 .73111395477

14.11101 .86784771721

14.21084 .00458147965

14.31066 .14131524209

14.41048 .27804900453

14.51030 .41478276698

$14.6 \quad 1012.82638347107$

14.7995 .447432264067

14.8978 .068481057064

14.9960 .689529850061

15.0943 .310578643057
15.1925 .931627436054

15.2908 .552676229051

15.3891 .756861059250

15.4875 .680716012216

15.5859 .604570965183

$15.6 \quad 843.528425918149$

15.7827 .452280871115

$15.8 \quad 811.376135824081$

15.9795 .299990777048

16.0779 .554826592807

16.1765 .102430182768

16.2750 .650033772729

16.3736 .197637362691

16.4721 .745240952652

16.5707 .292844542612

$16.6 \quad 692.840448132574$

16.7678 .388051722535

16.8665 .418872404800

16.9652 .740128720984

$17.0 \quad 640.061385037168$

$17.1 \quad 627.382641353352$

$17.2 \quad 614.703897669536$

$17.3 \quad 602.025153985720$

17.4589 .346410301904

$17.5 \quad 577.127415357433$

$17.6 \quad 565.781904687268$

17.7554 .436394017103

17.8543 .090883346938

17.9531 .745372676773

18.0520 .399862006608 


\section{Magnetic Field on Midplane of Booster Dipole}

\begin{tabular}{|c|c|c|}
\hline $18.1 \quad 509.054351336444$ & 20.1321 .023568964196 & 22.1171 .435711669852 \\
\hline $18.2 \quad 497.708840666279$ & $20.2 \quad 312.868437718537$ & $22.2 \quad 164.498603596021$ \\
\hline 487.334395320800 & $20.3 \quad 304.713306472877$ & $22.3 \quad 157.803943701659$ \\
\hline $.4 \quad 477.319840413637$ & 20.4296 .558175227218 & 22.4151 .171404419460 \\
\hline 467.305285506473 & $20.5 \quad 288.403043981558$ & $22.5 \quad 144.538865137261$ \\
\hline 457.290730599310 & 20.6280 .333956719487 & $22.6 \quad 137.906325855062$ \\
\hline 447.276175692147 & 20.7272 .789337109045 & $22.7 \quad 131.273786572862$ \\
\hline 437.261620784984 & $20.8 \quad 265.244717498604$ & 22.8124 .641247290663 \\
\hline 427.247065877821 & 20.9257 .700097888161 & $22.9 \quad 118.008708008464$ \\
\hline 417.232510970657 & $21.0 \quad 250.155478277719$ & 23.0111 .376168726265 \\
\hline 408.242840757854 & 21.1242 .610858667277 & 23.1104 .854869498242 \\
\hline .2399 .262961160166 & $21.2 \quad 235.066239056835$ & 23.298 .4763899492302 \\
\hline .3390 .283081562478 & $21.3 \quad 227.521619446394$ & 23.392 .0979104002185 \\
\hline 4381.303201964790 & $21.4 \quad 219.995468186664$ & 23.485 .7194308512066 \\
\hline 5372.323322367102 & 21.5213 .058360112834 & $23.5 \quad 79.3409513021949$ \\
\hline .6363 .343442769414 & $21.6 \quad 206.121252039004$ & 23.672 .9624717531829 \\
\hline .7354 .363563171726 & $21.7 \quad 199.184143965173$ & 23.766 .5839922041710 \\
\hline .8345 .488962701174 & $21.8 \quad 192.247035891343$ & $23.8 \quad 60.2055126551592$ \\
\hline .9337 .333831455515 & $21.9 \quad 185.309927817512$ & 23.953 .8270331061473 \\
\hline .0329 .178700209855 & $22.0 \quad 178.372819743682$ & $24.0 \quad 47.5693325627023$ \\
\hline & & 24.141 .3172123640681 \\
\hline & & 24.235 .0650921654340 \\
\hline & & $24.3 \quad 28.8129719668001$ \\
\hline & & 24.422 .5608517681659 \\
\hline & & $24.5 \quad 16.3087315695320$ \\
\hline & & 24.610 .0566113708978 \\
\hline & & 24.73 .80449117226369 \\
\hline
\end{tabular}

\section{LTB Centerline in the C5 Dipole}

The position of the LTB (Linac To Booster) centerline in the C5 dipole is given by Bleser [1] and is shown in Figure 4. Here the beam direction is from left to right. The dotted violet line is the centerline; it's angle with respect to the $x$ axis is $\theta_{4}=11.88573$ degrees. The equation of the line is

$$
y=m x+b
$$


where

$$
m=-\tan \theta_{4}, \quad b=13.939951 \mathrm{~m} .
$$

The $x$ and $y$ coordinates of the entrance to the dipole along the centerline are defined to be

$$
x_{I}=-\left\{\rho_{0}+W_{L} / 2\right\} \sin \left(\theta_{B} / 2\right), \quad y_{I}=m x_{I}+b
$$

where $W_{L}=0.762 \mathrm{~m}$ is the width of the dipole lamination.

Note that the LTB centerline intersects the inside wall of the vacuum chamber 10.7 inches upstream of the downstream end of the C5 magnet iron. Other possible neutral beam trajectories in the C5 dipole are shown in Figure 5. Here again the dotted violet line is the centerline. At the upstream end of the magnet, the solid violet lines are displaced $20 \mathrm{~mm}$ from the dotted line. The lower (upper) line has angle $-2(+2) \mathrm{mrad}$ with respect to the dotted line. These lines intersect the inside wall of the vacuum chamber 17.1 and 3.6 inches upstream of the downstream end of the magnet iron. This gives some idea of where neutral hydrogen atoms coming down the LTB line may hit the C5 vacuum chamber.

\section{H-minus Trajectory by Numerical Integration}

Let

$$
Z_{1}=x, \quad Z_{2}=\frac{d x}{d s}=x^{\prime}, \quad Z_{3}=y, \quad Z_{4}=\frac{d y}{d s}=y^{\prime}
$$

where $s$ is the distance along the H-minus trajectory and the primes denote differentiation with respect to $s$. Then the equations of motion on the midplane are [2]

$$
Z_{1}^{\prime}=Z_{2}, \quad Z_{2}^{\prime}=-K Z_{4}, \quad Z_{3}^{\prime}=Z_{4}, \quad Z_{4}^{\prime}=K Z_{2}
$$

where

$$
Z_{2}^{2}+Z_{4}^{2}=1
$$

and

$$
K=\frac{e}{c p} B\left(Z_{1}, Z_{3}\right)=\frac{e}{c p} B(x, y)=\frac{1}{\rho_{c}}\left\{B(x, y) / B_{c}\right\} .
$$

Here $e$ is the proton charge, $p$ is the particle momentum, $B(x, y)$ is the field on the midplane, $B_{c}$ is the field at the center of the magnet and

$$
\rho_{c}=\frac{c p}{e B_{c}} \text {. }
$$


The ratio $B(x, y) / B_{c}$ is given by Meng's data. These equations can be integrated numerically to obtain the particle trajectory. Employing the fourth-order Runge-Kutta method $[3,4]$ we obtain the trajectory shown in Figure 6. Here the H-minus beam enters the C5 dipole along the LTB centerline. The field in the channel through the backleg is assumed to be zero, so the trajectory follows the LTB centerline until it enters the region between the pole and backleg. It then follows the curved path shown in blue in the figure. Here we have assumed that $p$ and $B_{c}$ are such that

$$
\rho_{c}=\rho_{0}=L / \theta_{B}
$$

The blue curve ends at the magnet exit defined by the line

$$
\theta=\theta_{B} / 2=\pi / 36
$$

Below this line the field is assumed to be zero. At the magnet exit we find

$$
x=1.2060 \mathrm{~m}, \quad y=13.7844 \mathrm{~m}
$$

and

$$
x^{\prime}=0.99594, \quad y^{\prime}=-0.090024 .
$$

\section{Transport to the Injection Foil}

At the C5 dipole exit we transform to coordinates appropriate for the $\mathrm{C} 6$ straight section. The centerline of the straight is perpendicular to the line $\theta=\theta_{B} / 2$ and the transformation is

$$
x_{s}=C x-S y, \quad y_{s}=S x+C y-\rho_{0}
$$

where

$$
C=\cos \left(\theta_{B} / 2\right), \quad S=\sin \left(\theta_{B} / 2\right) .
$$

Using (16) we then have at the magnet exit

$$
x_{s}=0, \quad y_{s}=-28.48 \mathrm{~mm} \text {. }
$$

We also have

$$
\begin{aligned}
& \frac{d x_{s}}{d x}=C-S \frac{d y}{d x}=C-S\left(y^{\prime} / x^{\prime}\right) \\
& \frac{d y_{s}}{d x}=S+C \frac{d y}{d x}=S+C\left(y^{\prime} / x^{\prime}\right)
\end{aligned}
$$


and

$$
\frac{d y_{s}}{d x_{s}}=\frac{S x^{\prime}+C y^{\prime}}{C x^{\prime}-S y^{\prime}}=-2.8796 \text { mrad. }
$$

The drift length from the exit of the $\mathrm{C} 5$ dipole to the H-minus injection foil in the C6 straight is

$$
L_{F}=0.2726 \mathrm{~m}
$$

as given by Bleser [1]. Tracking the H-minus trajectory through the drift gives position and angle (with respect to the C6 centerline)

$$
X_{F}=y_{s}+L_{F} \frac{d y_{s}}{d x_{s}}=-29.3 \mathrm{~mm}, \quad X_{F}^{\prime}=\frac{d y_{s}}{d x_{s}}=-2.88 \mathrm{mrad}
$$

at the injection foil.

Now let $X_{0}$ and $X_{0}^{\prime}$ be the position and angle of an H-minus particle with respect to the LTB centerline at the entrance to the C5 dipole. Tracking the particle through the $\mathrm{C} 5$ dipole as in the previous section and then tracking through the drift to the foil we obtain the positions and angles listed in Table 1.

Table 1: Position and Angle at Injection Foil obtained by numerical integration with various values of $X_{0}$ and $X_{0}^{\prime}$. Units are mm and mrad.

\begin{tabular}{|c|c|c|c|}
\hline$X_{0}$ & $X_{0}^{\prime}$ & $X_{F}$ & $X_{F}^{\prime}$ \\
\hline 10 & 0 & -33.5 & 0.20 \\
0 & 5 & -40.6 & -6.51 \\
\hline 0 & 0 & -29.3 & -2.88 \\
\hline 0 & -5 & -18.0 & 0.74 \\
-10 & 0 & -25.0 & -5.95 \\
\hline
\end{tabular}

\section{Dispersion at Injection Foil}

The calculations of the previous two sections were carried out with $\rho_{c} / \rho_{0}=1$. If we carry them out with $\rho_{c} / \rho_{0}$ equal to various values close to 1 then we can obtain the dispersion of the H-minus beam at the injection foil. We have

$$
\rho_{c}=\frac{c p}{e B_{c}}, \quad \rho_{0}=\frac{c p_{0}}{e B_{c}}=L / \theta_{B}
$$


and

$$
\rho_{c} / \rho_{0}-1=p / p_{0}-1=\frac{p-p_{0}}{p_{0}}=\frac{\Delta p}{p_{0}} .
$$

Table 2 lists the positions $X_{F}$ and angles $X_{F}^{\prime}$ obtained at the injection foil for various values of $\Delta p / p_{0}$.

Table 2: Position and Angle at Injection Foil obtained by numerical integration with various values of $\Delta p / p_{0}$.

\begin{tabular}{|c|c|c|c|}
\hline$\rho_{c} / \rho_{0}$ & $\Delta p / p_{0}$ & $X_{F}(\mathrm{~mm})$ & $X_{F}^{\prime}(\mathrm{mrad})$ \\
\hline 1.020 & 0.020 & -31.8 & -5.16 \\
1.015 & 0.015 & -31.1 & -4.60 \\
1.010 & 0.010 & -30.5 & -4.03 \\
1.005 & 0.005 & -29.9 & -3.45 \\
\hline 1.000 & 0.000 & -29.3 & -2.88 \\
\hline 0.995 & -0.005 & -28.6 & -2.28 \\
0.990 & -0.010 & -28.0 & -1.69 \\
0.985 & -0.015 & -27.3 & -1.09 \\
0.980 & -0.020 & -26.7 & -0.48 \\
\hline
\end{tabular}

Here we see that for $\Delta p / p_{0}=0.01$ we have

$$
\Delta X_{F}=-1.27 \mathrm{~mm}, \quad \Delta X_{F}^{\prime}=-1.17 \mathrm{mrad} .
$$

This gives the dispersion at the foil due to transport of H-minus beam through the C5 dipole.

\section{Hard Edge Approximation}

Consider the region on the midplane with radial and azimuthal coordinates such that

$$
\rho_{0}-H \leq r \leq \rho_{0}+H, \quad-\theta_{B} / 2 \leq \theta \leq \theta_{B} / 2
$$

where

$$
\rho_{0}=L / \theta_{B}, \quad H=W_{P} / 2+d, \quad \theta_{B}=2 \pi / 36 .
$$

Here $W_{P}=254 \mathrm{~mm}$ is the pole width and the pole edges are at radii $r=\rho_{0} \pm W_{P} / 2$. The parameter $d$ gives the radial distance from the pole edges to the limits $r=\rho_{0} \pm H$. As an approximation we assume that the 
field everywhere in this region is equal to the field $B_{c}$ at the center of the magnet. The radius-of-curvature for particle motion in the region is

$$
\rho_{c}=\frac{c p}{e B_{c}}
$$

where $p$ is the particle momentum. Outside the region the field is assumed to be zero. The radii $r=\rho_{0} \pm H$ define the hard edges of the field. Under these assumptions the incoming H-minus beam follows the LTB centerline until it encounters the hard edge of the field at radial coordinate $r=\rho_{0}+H$. At this point the beam begins to follow a circular arc with radius-of-curvature $\rho_{c}$. This continues until the beam reaches the magnet exit defined by the line

$$
\theta=\theta_{B} / 2=\pi / 36 .
$$

Below this line the field is zero and the beam follows a straight line to the injection foil.

Taking $\rho_{c}=\rho_{0}$ and $d=37.7 \mathrm{~mm}$ we obtain the trajectory shown in

Figure 7. Here the dotted blue curves show the hard edges of the field.

The drift length along the LTB centerline from the magnet entrance to the hard edge at $r=\rho_{0}+H$ is $\lambda=858.251 \mathrm{~mm}$. At the intersection of the centerline with the hard edge, the trajectory has angle $\psi=76.4731$ degrees with respect to the line perpendicular to the hard edge as shown in Figure 8. This results in horizontal focusing (not defocusing!) of the H-minus beam. The trajectory follows the circular arc shown in blue in Figure 7. The turning angle of the arc is $\phi=6.72156$ degrees. Tracking the trajectory through the drift between the magnet exit and the injection foil gives position and angle

$$
X_{F}=-30.2 \mathrm{~mm}, \quad X_{F}^{\prime}=-2.87 \mathrm{mrad}
$$

at the foil. Comparing these values with those given in (25), we see that the hard edge approximation with $d=37.7 \mathrm{~mm}$ gives essentially the same trajectory as that obtained by numerical integration of the equations of motion using the field calculated by Meng. Table 3 lists the parameters $\lambda$, $\psi, \phi, X_{F}$, and $X_{F}^{\prime}$ obtained for various values of the hard edge parameter $d$.

Figure 9 shows the trajectory of a proton which enters the C5 dipole along the LTB centerline. The proton travels along the centerline until it encounters the hard edge of the field. At this point it begins to follow a circular arc with radius-of-curvature $\rho_{c}$. Taking $\rho_{c}=\rho_{0}$ and hard edge 
parameter $d=37.7 \mathrm{~mm}$ we obtain the magneta curve shown in the figure. This curve intersects the inside wall of the vacuum chamber 21.0 inches upstream of the downstream end of the C5 magnet iron. This gives some idea of where protons coming down the LTB line may hit the C5 vacuum chamber.

Table 3: Parameters obtained using Hard Edge Approximation.

\begin{tabular}{|c|c|c|c|c|c|}
\hline$d(\mathrm{~mm})$ & $\lambda(\mathrm{mm})$ & $\psi$ & $\phi$ & $X_{F}(\mathrm{~mm})$ & $X_{F}^{\prime}(\mathrm{mrad})$ \\
\hline 30 & 891.328 & 76.6045 & 6.58586 & -34.6554 & -5.23372 \\
\hline 31 & 887.014 & 76.5874 & 6.60356 & -34.0758 & -4.92483 \\
32 & 882.706 & 76.5703 & 6.62123 & -33.4956 & -4.61634 \\
33 & 878.403 & 76.5532 & 6.63889 & -32.9148 & -4.30824 \\
34 & 874.105 & 76.5361 & 6.65652 & -32.3334 & -4.00052 \\
\hline 35 & 869.813 & 76.5190 & 6.67413 & -31.7514 & -3.69318 \\
\hline 36 & 865.526 & 76.5020 & 6.69171 & -31.1688 & -3.38623 \\
37 & 861.245 & 76.4850 & 6.70928 & -30.5856 & -3.07965 \\
37.7 & 858.251 & 76.4731 & 6.72156 & -30.1771 & -2.86528 \\
38 & 856.968 & 76.4680 & 6.72682 & -30.0019 & -2.77346 \\
39 & 852.697 & 76.4511 & 6.74434 & -29.4176 & -2.46764 \\
\hline 40 & 848.431 & 76.4341 & 6.76185 & -28.8327 & -2.16220 \\
\hline 41 & 844.171 & 76.4172 & 6.77932 & -28.2472 & -1.85713 \\
42 & 839.915 & 76.4003 & 6.79678 & -27.6612 & -1.55244 \\
43 & 835.665 & 76.3835 & 6.81422 & -27.0746 & -1.24811 \\
44 & 831.420 & 76.3666 & 6.83163 & -26.4874 & -0.94416 \\
\hline 45 & 827.180 & 76.3498 & 6.84903 & -25.8997 & -0.64057 \\
\hline
\end{tabular}

\section{Linear Matrix for Transport of H-minus Beam through the C5 Dipole}

The hard edge approximation allows us to write down a linear matrix for the transport of H-minus beam through the C5 dipole. In the horizontal plane the transfer matrix is [5]

$$
\mathbf{M}=\left(\begin{array}{lll}
M_{11} & M_{12} & M_{13} \\
M_{21} & M_{22} & M_{23} \\
M_{31} & M_{32} & M_{33}
\end{array}\right)=\mathbf{B Q L}
$$


where

$$
\begin{gathered}
\mathbf{B}=\left(\begin{array}{ccc}
C & \rho S & D \\
-S / \rho & C & D^{\prime} \\
0 & 0 & 1
\end{array}\right), \quad C=\cos \phi, \quad S=\sin \phi \\
D=\rho(1-\cos \phi), \quad D^{\prime}=\sin \phi
\end{gathered}
$$

and

$$
\mathbf{Q}=\left(\begin{array}{ccc}
1 & 0 & 0 \\
-T / \rho & 1 & 0 \\
0 & 0 & 1
\end{array}\right), \quad \mathbf{L}=\left(\begin{array}{lll}
1 & \lambda & 0 \\
0 & 1 & 0 \\
0 & 0 & 1
\end{array}\right), \quad T=\tan \psi
$$

Here we take hard edge parameter $d=37.7$ as before. This gives $\phi=6.72156$ degrees, $\psi=76.4731$ degrees, and $\lambda=0.858251 \mathrm{~m}$. The radius-of-curvature is $\rho=\rho_{0}=13.8656 \mathrm{~m}$. Note that the effect of the angle $\psi$ at the hard edge of the field is to focus the beam in the horizontal plane. Carrying out the matrix multiplications we have

$$
\begin{gathered}
M_{11}=C-T S, \quad M_{12}=\rho S+\lambda(C-T S), \quad M_{13}=D \\
M_{21}=-(C T+S) / \rho, \quad M_{22}=C-\lambda(C T+S) / \rho, \quad M_{23}=D^{\prime}
\end{gathered}
$$

and

$$
M_{31}=0, \quad M_{32}=0, \quad M_{33}=1 .
$$

Putting in numbers we have

$$
C=0.993127, \quad S=0.117044, \quad T=4.15670
$$

and

$$
D=0.095303 \mathrm{~m}, \quad D^{\prime}=0.117044
$$

In the vertical plane the transfer matrix is

$$
\mathbf{N}=\mathbf{A Q}^{-1} \mathbf{L}
$$

where

$$
\mathbf{A}=\left(\begin{array}{ccc}
1 & \rho \phi & 0 \\
0 & 1 & 0 \\
0 & 0 & 1
\end{array}\right)
$$

Here the effect of the angle $\psi$ at the hard edge of the field is to defocus the beam. 


\section{Transport to Foil using Linear Matrix}

The linear matrix for the transport of H-minus beam from the C5 dipole entrance to the injection foil is

$$
\left(\begin{array}{ccc}
T_{11} & T_{12} & T_{13} \\
T_{21} & T_{22} & T_{23} \\
T_{31} & T_{32} & T_{33}
\end{array}\right)=\left(\begin{array}{ccc}
1 & L_{F} & 0 \\
0 & 1 & 0 \\
0 & 0 & 1
\end{array}\right)\left(\begin{array}{lll}
M_{11} & M_{12} & M_{13} \\
M_{21} & M_{22} & M_{23} \\
M_{31} & M_{32} & M_{33}
\end{array}\right)
$$

where

$$
L_{F}=0.2726 \mathrm{~m}
$$

is the drift length from the dipole exit to the injection foil. The elements $M_{i j}$ are given by (38-40). Carrying out the matrix multiplication we have

$$
\begin{gathered}
T_{11}=M_{11}+L_{F} M_{21}, \quad T_{12}=M_{12}+L_{F} M_{22}, \quad T_{13}=D+L_{F} D^{\prime} \\
T_{21}=M_{21}, \quad T_{22}=M_{22}, \quad T_{23}=D^{\prime} \\
T_{31}=0, \quad T_{32}=0, \quad T_{33}=1 .
\end{gathered}
$$

Let $X_{0}$ and $X_{0}^{\prime}$ be the position and angle of an H-minus particle with respect to the LTB centerline at the C5 dipole entrance. Let $\Delta p$ be the deviation of the particle's momentum from the nominal momentum $p_{0}$ given by (26). Then the position and angle (with respect to the C6 straight section centerline) at the foil are

$$
\begin{aligned}
& X_{F}=X_{F 0}-T_{11} X_{0}-T_{12} X_{0}^{\prime}-T_{13} \Delta p / p_{0} \\
& X_{F}^{\prime}=X_{F 0}^{\prime}-T_{21} X_{0}-T_{22} X_{0}^{\prime}-T_{23} \Delta p / p_{0}
\end{aligned}
$$

where $X_{F 0}$ and $X_{F 0}^{\prime}$ are the nominal position and angle given by (33).

Table 4 lists the values of $X_{F}$ and $X_{F}^{\prime}$ obtained from (50) and (51) for various values of $X_{0}$ and $X_{0}^{\prime}$ with $\Delta p / p_{0}=0$. These numbers are in good agreement with those obtained by numerical integration and listed in

Table 1.

For the case in which $X_{0}=0, X_{0}^{\prime}=0$, and $\Delta p / p_{0}=0.01$ we obtain

$$
\Delta X_{F}=X_{F}-X_{F 0}=-1.27 \mathrm{~mm}
$$

and

$$
\Delta X_{F}^{\prime}=X_{F}^{\prime}-X_{F 0}^{\prime}=-1.17 \mathrm{mrad}
$$

which give the dispersion at the foil due to transport of H-minus beam through the C5 dipole. These numbers are in good agreement with those obtained by numerical integration and listed in (28). 
Table 4: Position and Angle at Injection Foil obtained using linear transfer matrix with various values of $X_{0}$ and $X_{0}^{\prime}$. Units are $\mathrm{mm}$ and mrad.

\begin{tabular}{|c|c|c|c|}
\hline$X_{0}$ & $X_{0}^{\prime}$ & $X_{F}$ & $X_{F}^{\prime}$ \\
\hline 10 & 0 & -34.4 & 0.20 \\
0 & 5 & -41.5 & -6.52 \\
\hline 0 & 0 & -30.2 & -2.87 \\
\hline 0 & -5 & -18.9 & 0.79 \\
-10 & 0 & -25.9 & -5.93 \\
\hline
\end{tabular}

\section{Acknowledgement}

I would like to thank Wuzheng Meng for his calculations of the field on the midplane of the Booster dipole.

\section{References}

[1] E. Bleser, "Geometry of the Booster Injection Region", Booster Technical Note 216, January 6, 1993

[2] C.J. Gardner, "Notes on Orbit Equations in the AGS", C-A/AP/Note 164, September, 2004, p. 3

[3] W.H. Press, et al, "Numerical Recipes (Fortran Version)", Cambridge University Press, 1989, pp. 550-554.

[4] N. Gershenfeld, "The Nature of Mathematical Modeling", Cambridge University Press, 1999, pp. 67-72.

[5] D.C. Carey, "The Optics of Charged Particle Beams, Harwood Academic Publishers, New York, 1987. 


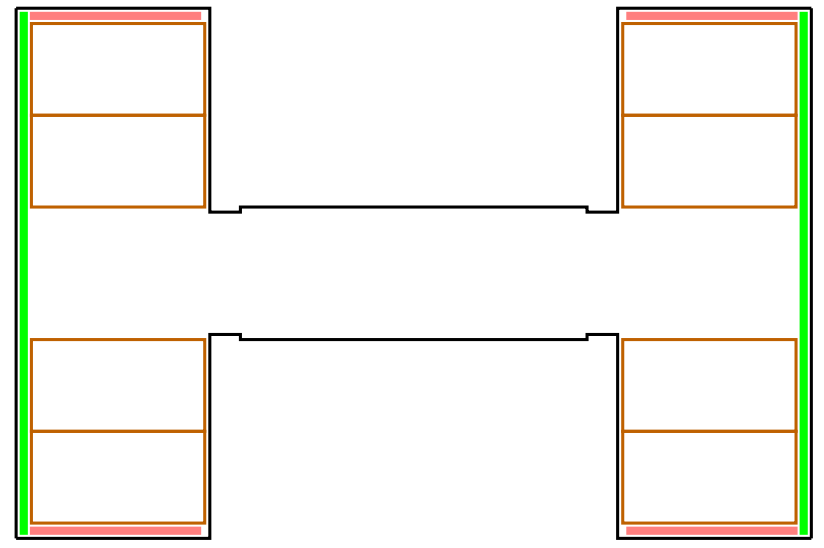

Figure 1: Scale drawing of Booster dipole lamination. The pole width is $254 \mathrm{~mm}$. The distance from the pole to the backleg is $121 \mathrm{~mm}$. The width of the backleg is $133 \mathrm{~mm}$. The vertical gap between the poles is $82.55 \mathrm{~mm}$. The shims on the poles are $19 \mathrm{~mm}$ wide and the vertical gap between them is $77.75 \mathrm{~mm}$. The height of the lamination is $596.55 \mathrm{~mm}$ and the height of the H-shaped opening is $330.55 \mathrm{~mm}$. The brown rectangles indicate magnet coils. G10 insulating strips are indicated by the thin green rectangles. The thin pink rectangles indicate trim windings. 


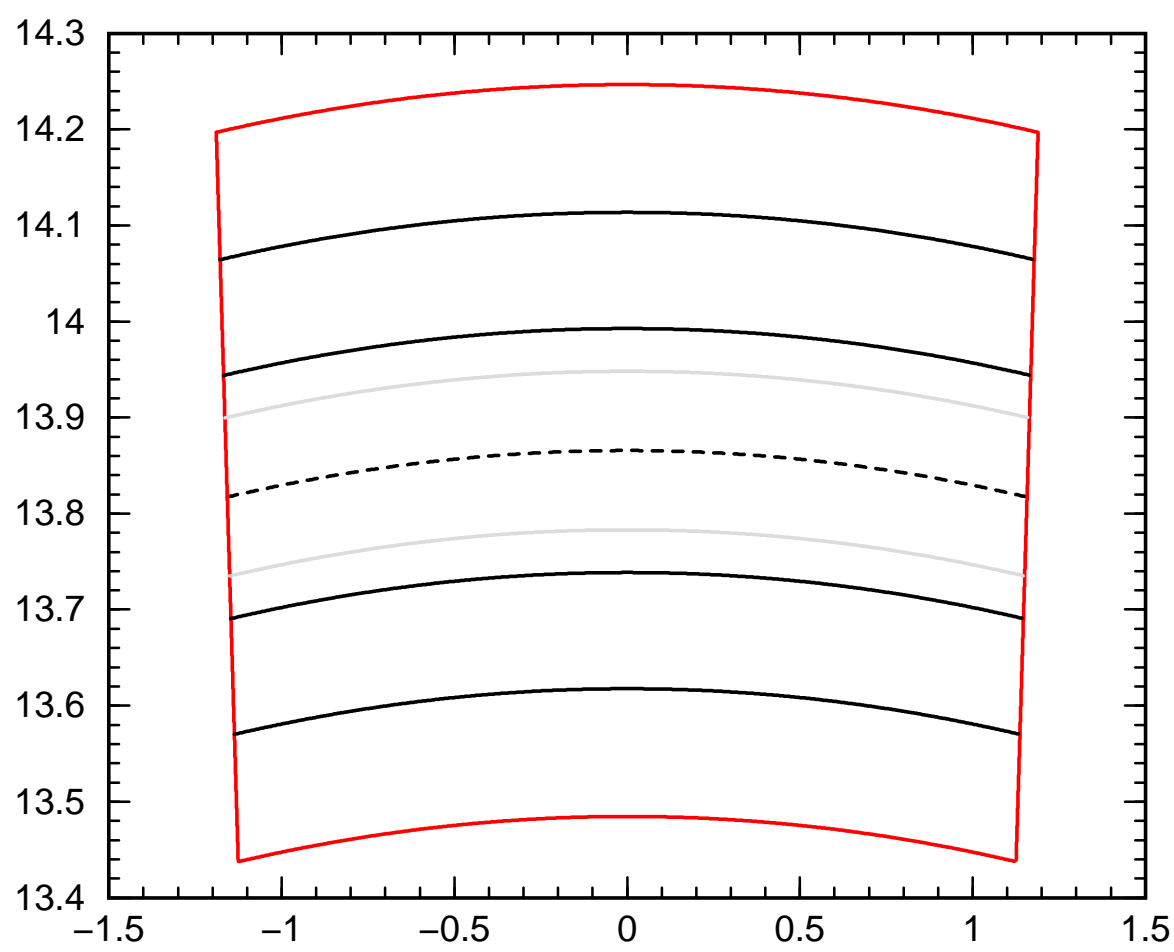

Figure 2: Top view of Booster Dipole. Beam direction is from left to right. The horizontal and vertical axes give the $x$ and $y$ coordinates on the magnet midplane. The units are meters. The radial and azimuthal coordinates are defined by $x=r \sin \theta$ and $y=r \cos \theta$. The line $x=0$ bisects the magnet. The magnetic length is $L=2.42 \mathrm{~m}$ and the bend angle is $\theta_{B}=2 \pi / 36$. The dotted black curve is the nominal trajectory of circulating beam on the midplane; it is a circular arc with radius-of-curvature $\rho=L / \theta_{B}=13.8656$ $\mathrm{m}$. The center of curvature is the point $x=0, y=0$. As noted by Bleser [1] the centerline of the dipole is actually displaced radially outward by 0.18 $\mathrm{mm}$ with respect to the nominal trajectory. The practical consequences of this are negligible and we assume that the centerline and nominal trajectory coincide. The red lines and curves show the perimeter of the magnet iron. The left and right edges of the magnet iron lie on the lines $\theta=\mp \theta_{2} / 2$, where $\theta_{2}=9.5763$ degrees as given by Bleser [1]. The gray curves are the projections of the walls of the vacuum chamber onto the midplane; these are $82.55 \mathrm{~mm}$ from the magnet centerline. The black curves are the projections of the pole and backleg boundaries. Those closest to the centerline mark the pole boundaries; they are $127 \mathrm{~mm}$ from the centerline. The outer black curves mark the backleg boundaries; these are $248 \mathrm{~mm}$ from the centerline. 


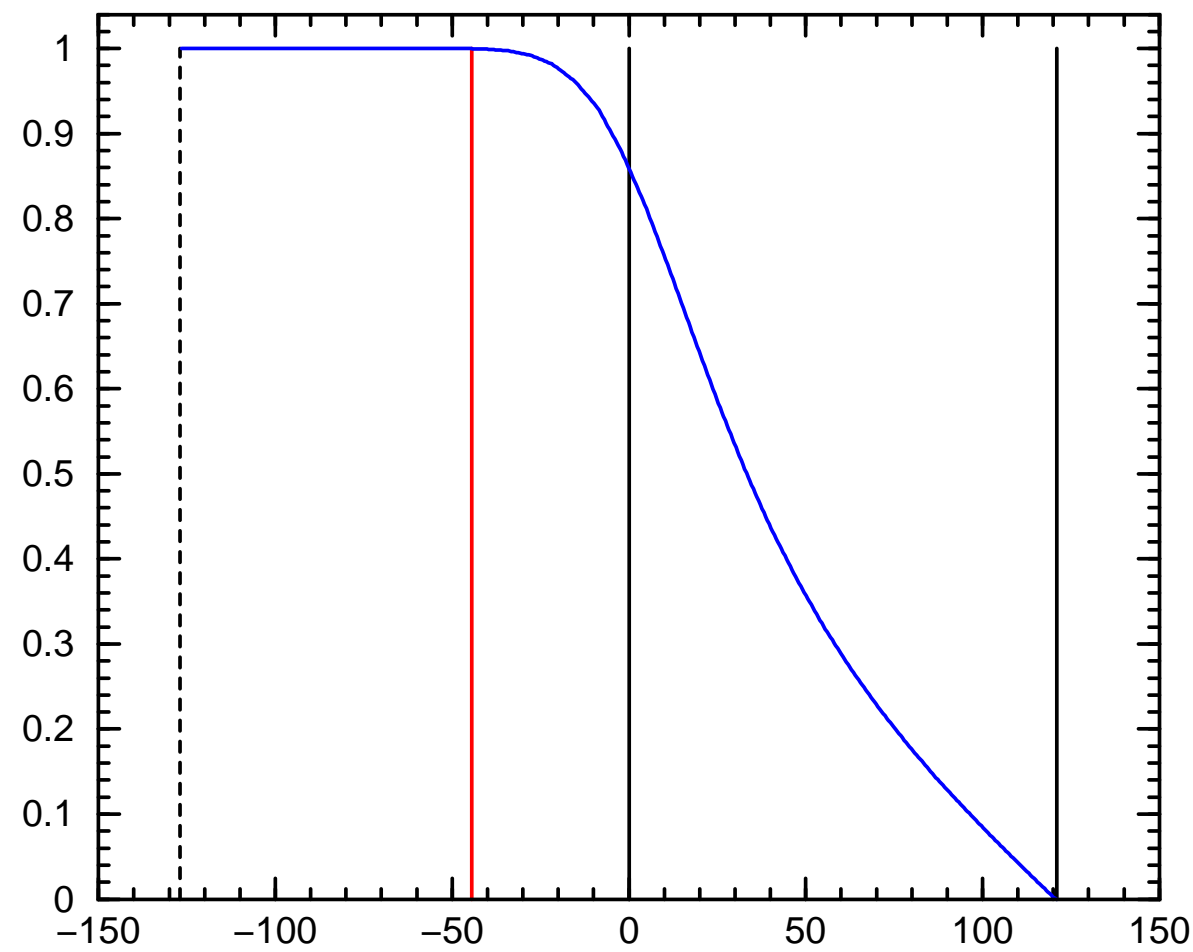

Figure 3: Magnetic field on the midplane obtained by Wuzheng Meng using the Opera code. The blue curve gives the field in units of the field $B_{c}$ at the center of the magnet. The horizontal axis gives the radial distance from the projection of the pole edge on the midplane. The units are $\mathrm{mm}$. The black lines at 0 and 121 mark the pole and backleg edges. The dotted line at $-127 \mathrm{~mm}$ marks the center of the magnet; the red line at $-44.45 \mathrm{~mm}$ marks the vacuum chamber wall. Numerical integration of the equations of motion using this field gives the trajectory of the H-minus beam on the midplane of the C5 dipole. 


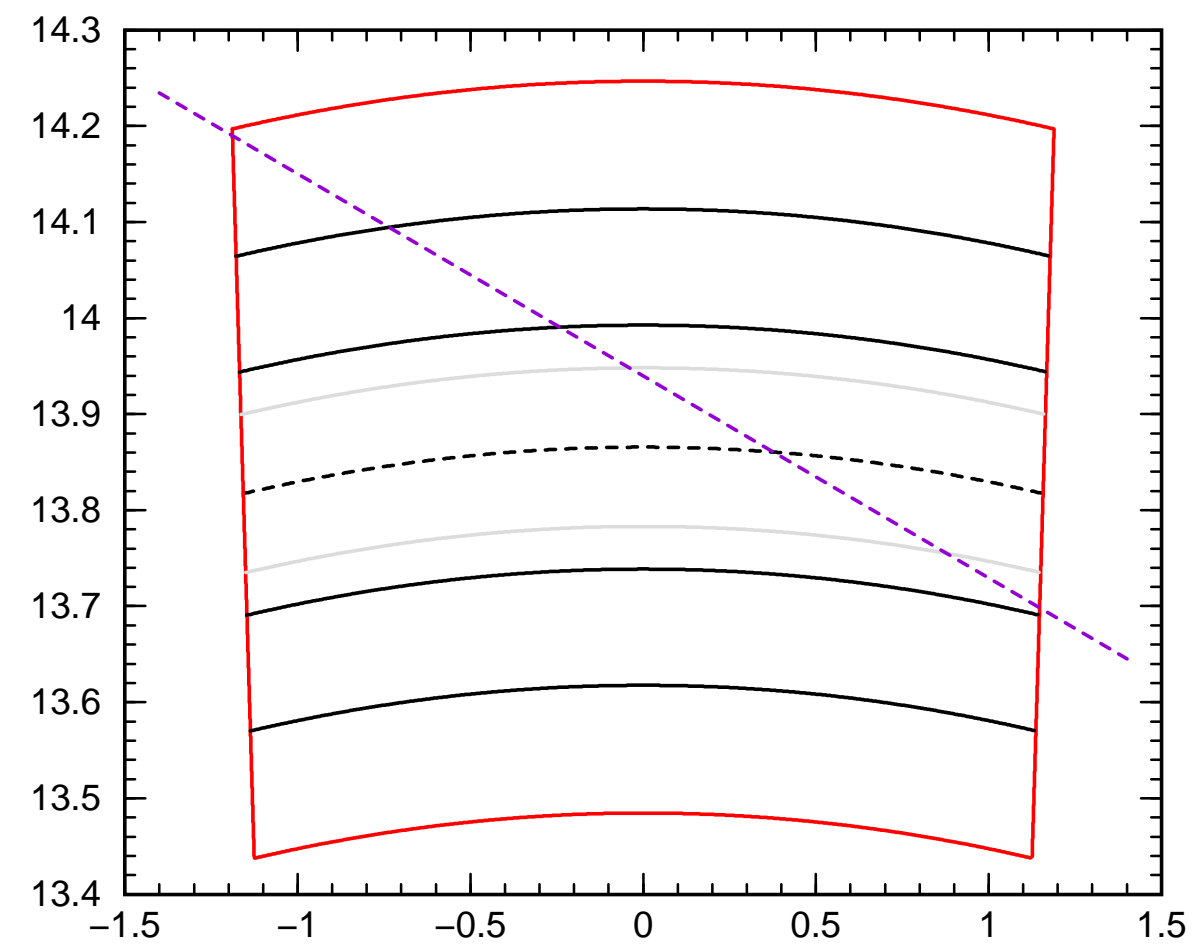

Figure 4: Position of the LTB centerline in the C5 Dipole as given by Bleser [1]. Here the beam direction is from left to right. The dotted violet line is the centerline; it's angle with respect to the $x$ axis is $\theta_{4}=11.88573$ degrees. The equation of the line is $y=m x+b$ where $m=-\tan \theta_{4}$ and $b=13.939951 \mathrm{~m}$. The $x$ and $y$ coordinates of the entrance to the dipole along the centerline are defined to be $x_{I}=-\left\{\rho_{0}+W_{L} / 2\right\} \sin \left(\theta_{B} / 2\right), y_{I}=m x_{I}+b$ where $W_{L}=0.762 \mathrm{~m}$ is the width of the dipole lamination. The centerline intersects the inside wall of the vacuum chamber 10.7 inches upstream of the downstream end of the C5 magnet iron. 


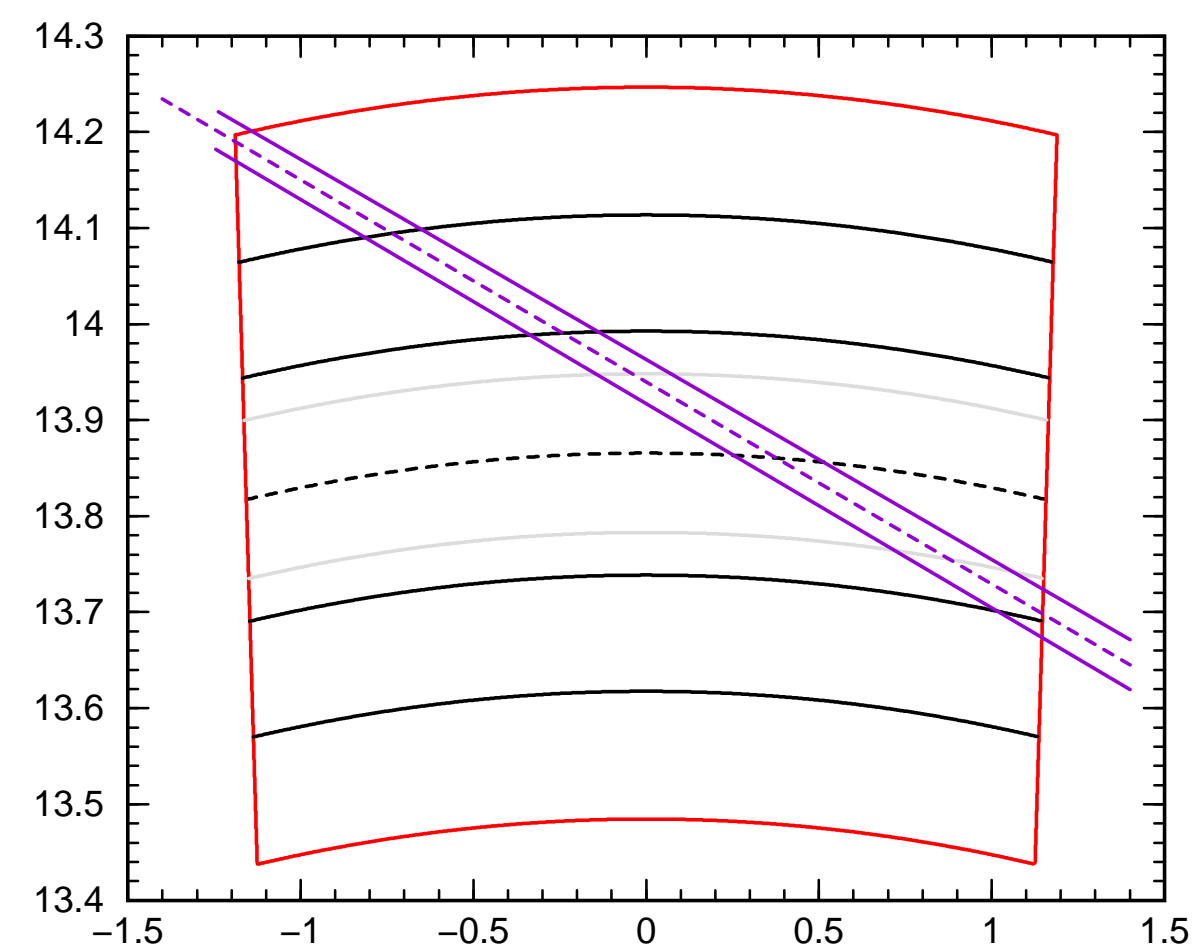

Figure 5: Neutral Beam Trajectories on the midplane. Here again the dotted violet line is the LTB centerline; it intersects the inside wall of the vacuum chamber 10.7 inches upstream of the downstream end of the C5 magnet iron. At the upstream end of the magnet, the solid violet lines are displaced 20 $\mathrm{mm}$ from the dotted line. The lower (upper) line has angle $-2(+2) \mathrm{mrad}$ with respect to the dotted line. These lines intersect the inside wall of the vacuum chamber 17.1 and 3.6 inches upstream of the downstream end of the magnet iron. This gives some idea of where neutral hydrogen atoms coming down the LTB line may hit the C5 vacuum chamber. 


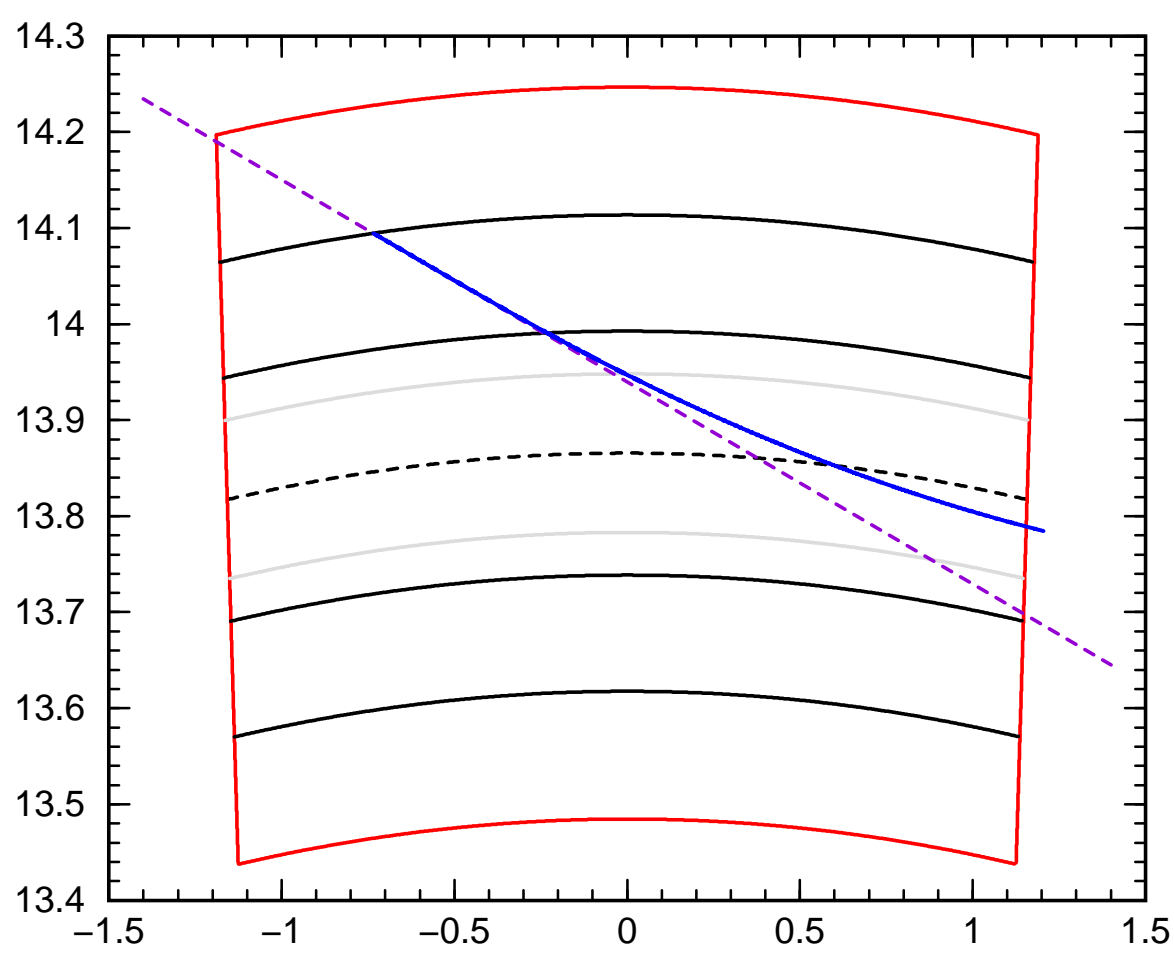

Figure 6: H-minus Trajectory on C5 Dipole Midplane. Here, as before, the dotted violet line is the LTB centerline. The blue curve is the H-minus trajectory obtained by numerical integration of the equations of motion using the field calculated by Meng. The field is assumed to be zero in the channel that passes through the backleg and is assumed to be independent of the azimuthal position on the midplane. The incoming H-minus beam travels along the LTB centerline until it enters the region between the pole and backleg where the field is nonzero. The blue curve ends at the magnet exit defined by the line $\theta=\theta_{B} / 2=\pi / 36$. Below this line the field is assumed to be zero. The drift length from the exit of the magnet to the H-minus injection foil in the C6 straight section is $0.2726 \mathrm{~m}$ as given by Bleser [1]. Tracking the trajectory through the drift gives position $-29.3 \mathrm{~mm}$ and angle $-2.88 \mathrm{mrad}$ (with respect to the C6 centerline) at the foil. 


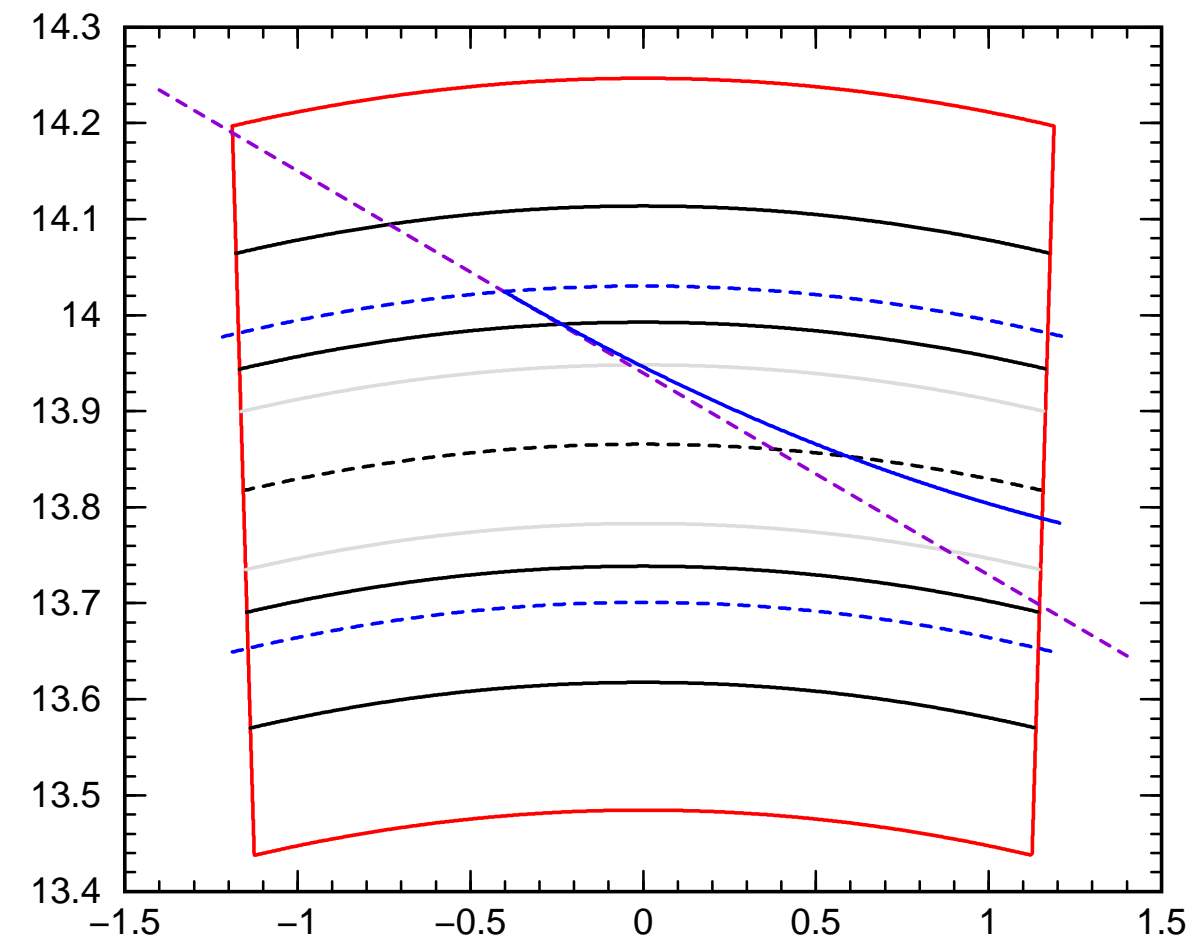

Figure 7: H-minus Trajectory on C5 Dipole Midplane. Here the hard edge approximation has been used to calculate the beam trajectory. The hard edges of the field are shown by the dotted blue curves. These are $d=37.7$ $\mathrm{mm}$ from the pole edges. The solid blue curve is the H-minus trajectory. The resulting position and angle of the H-minus beam at the injection foil are $-30.2 \mathrm{~mm}$ and $-2.87 \mathrm{mrad}$, in good agreement with the position and angle obtained by numerical integration. 


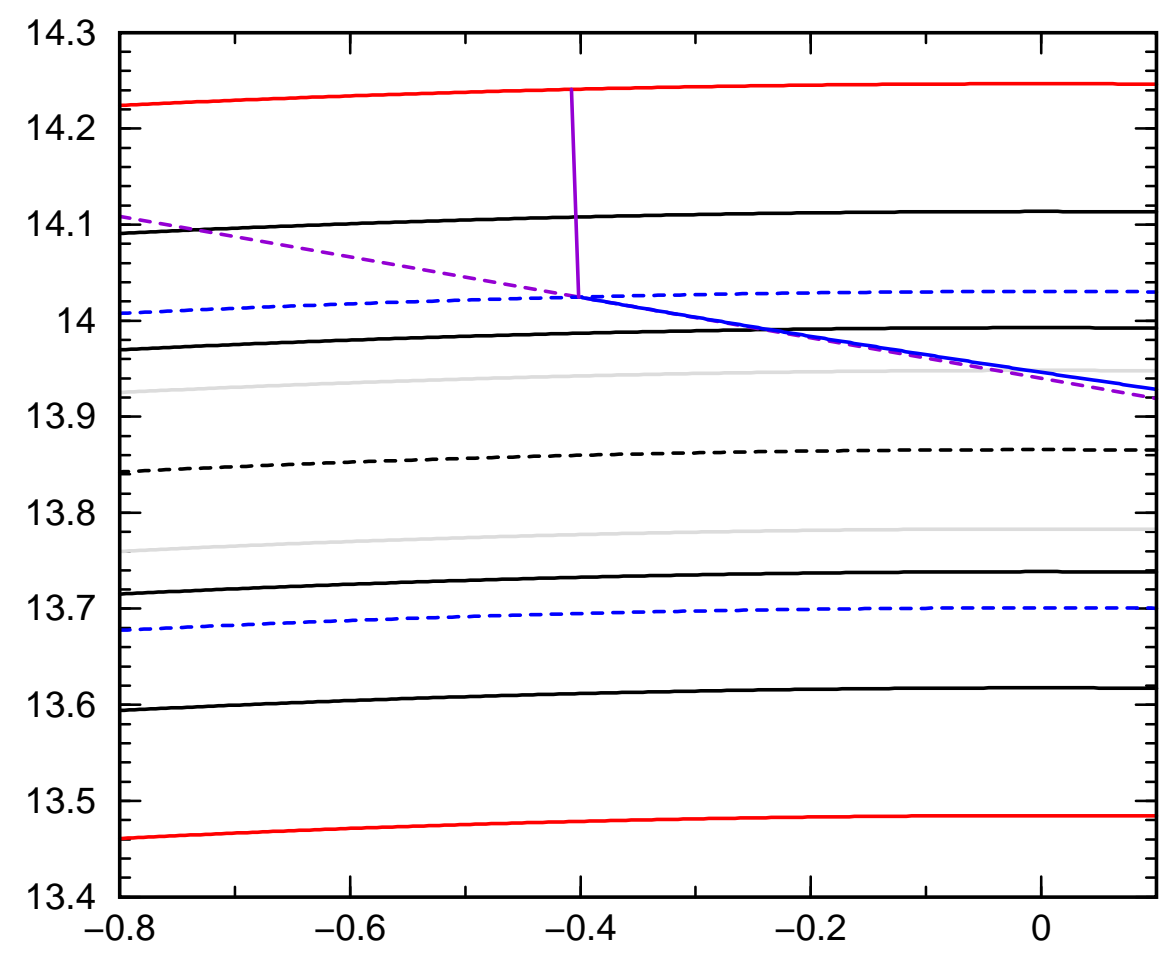

Figure 8: Expanded view of Figure 7. Here the solid violet line is perpendicular to the hard edge (dotted blue curve) of the field at the intersection of the centerline (dotted violet line) with the hard edge. The angle between the centerline and the solid violet line is $\psi=76.4731$ degrees. This results in horizontal focusing of the $\mathrm{H}$-minus beam. The focal length is $f=\rho / \tan \psi=3.34 \mathrm{~m}$. 


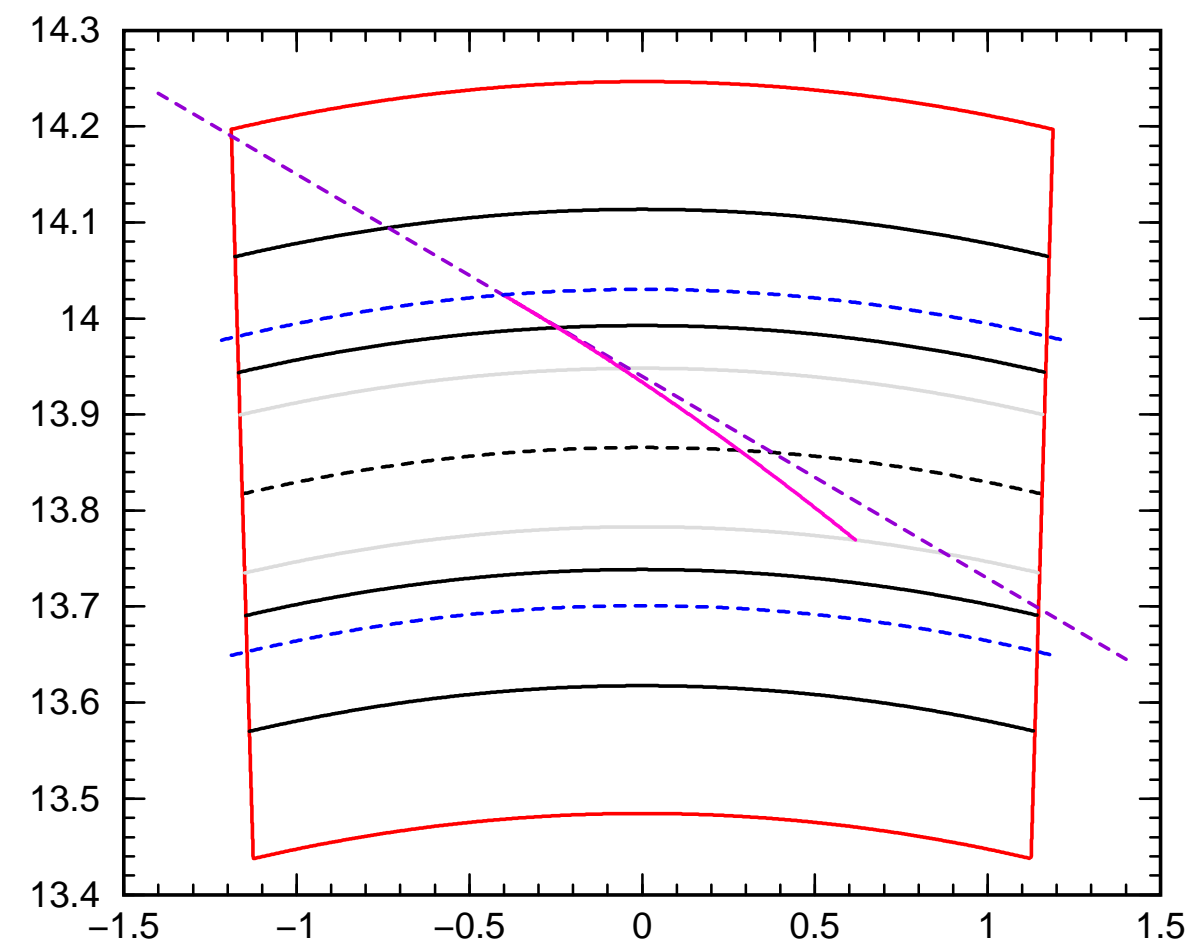

Figure 9: Proton Trajectory on C5 Dipole Midplane. Dotted violet line is the LTB center line. The solid magenta line is the proton trajectory obtained using the hard edge approximation with $d=37.7 \mathrm{~mm}$. The dotted blue curves show the hard edges of the field. The proton beam hits the inside wall of the vacuum chamber 21.0 inches upstream of the downstream end of the C5 magnet iron. 